\title{
ARTICLE
}

\section{The origin dependence of the material constants: the permittivity and the inverse permeability}

\author{
Marco Anelli ${ }^{a}$, Dan Jonsson ${ }^{a}$, Heike Fliegl $^{b}$ and Kenneth Ruud ${ }^{a *}$, \\ ${ }^{a}$ Center for Theoretical and Computational Chemistry (CTCC), Department of \\ Chemistry University of Tromsø, 9037 Tromsø, Norway; ${ }^{b}$ Center for Theoretical and \\ Computational Chemistry (CTCC), Department of Chemistry University of Oslo, \\ P.O.Box 1033 Blindern, 0315 Oslo, Norway;
}

(XX December 2014)

\begin{abstract}
New derivations of origin-independent expressions for the electric permittivity are presented, starting either from the response function of the current density that defines the absorption coefficient, or from the off-resonance single-photon scattering amplitude that leads to the Kramers-Heisenberg dispersion formula. The resulting expression for the permittivity is compared with earlier work on the origin dependence of the material constants. Different origin-independent expressions for the permittivity, the inverse permeability and the magnetizability are calculated and discussed. By considering electromagnetic plane waves in the absence of external sources, the macroscopic Maxwell equations are used to describe the response of matter to external fields. In combination with the constitutive relations, a wave equation expressed in terms of the material constants is derived. It is shown that the different definitions of the material constants lead to the same wave equation. The non-uniqueness of the definitions of the material constants is discussed in this context. Finally, based on the discussions, we propose a possible unique, origin-independent definition of the material constants.
\end{abstract}

Keywords: Material constants; frequency-dependent magnetizability; permittivity; inverse permeability; scattering amplitude; absorption coefficients

\section{Introduction}

The multipole description of electrodynamics has been widely used to derive expressions for molecular properties in order to describe physical phenomena such as reflection, scattering and other optical effects. [1] Multipole theory is commonly introduced in microscopic electrodynamics and extended to the macroscopic case through a spatial averaging process $[1,2]$. When using multipole theory, the expressions obtained for many molecular properties are origin dependent when going beyond the lowest non-zero multipole order, even if the observable quantities remain, by necessicity, origin independent. This is found for both microscopic and macroscopic theories. Origin dependence means that the respective properties explicitly depend on the choice of the origin of the coordinate system (see e.g. Refs. [1, 3]). It is therefore important to derive origin-independent expressions for observable molecular properties, but controversy remains [4-6].

Raab and de Lange proposed a transformation theory [7] that — when applied to the macroscopic description of electrodynamics - leads to origin-independent ex-

*Corresponding author. Email: kenneth.ruud@uit.no 
pressions for the material constants, also known as the constitutive tensors. This transformation theory makes use of the macroscopic Maxwell equations and exploits the non-uniqueness of the definition of the auxiliary fields $\boldsymbol{D}$ and $\boldsymbol{H}$. The theory provides satisfactory results in the sense that origin-independent expressions are obtained. However, limitations regarding their physical behaviour remain, since the material constants in these derivations are not directly related to experimental measurable quantities. In the following, we will relate the material constants to measurable quantities such as the scattering amplitude.

The magnetizability has often been at the core of the debate on gauge-origin independent expressions for molecular properties. The expression for the magnetizability obtained from multipole theory is origin independent for a static external magnetic field, but appears to be origin dependent in the frequency-dependent case. Raab and de Lange proposed an origin-independent definition of the frequency-dependent magnetizability in the microscopic case [8] that agrees with the macroscopic expression achieved for the inverse permeability using their transformation theory. The validity of this expression has still to be investigated, since an alternative definition of the inverse permeability can be derived in the macroscopic case [9]. Furthermore, the theory used to derive the magnetizability in the microscopic case relies on the invocation of a number of requirements that the frequency-dependent magnetizability is assumed to possess [8]. Although all the requirements imposed are reasonable, their introduction is nevertheless of an ad hoc nature.

In this work, the origin-independent expression for the permittivity obtained by Raab and de Lange is derived in a new way, using two alternative approaches: (1) by considering the expression for the off-resonance single-photon scattering amplitude [10], and (2) starting from the response function of the current density that defines the absorption coefficient [11]. It is shown that these procedures do not require any transformation theory, only a Taylor expansion (to second order) of the external electromagnetic perturbation. The results can be considered as a generalization, beyond the dipole approximation, of the work of Healy [10] on the Kramers-Heisenberg dispersion formula.

The macroscopic Maxwell equations and the constitutive relations [1] are then discussed, and it is shown that a general and unique definition of the material constants is still not established. Therefore, possible alternative expressions have to be considered. It is shown that a covariant formulation of the auxiliary fields and the constitutive relations leads to origin-independent expressions for the material constants [9, 12] that differ from the ones given in Ref. [7]. Particular attention is paid to the inverse permeability, noting that the result obtained for the electric permittivity suggests that transformed multipole theory provides correct and unique definitions of the material constants.

Next we consider the wave equation expressed in terms of the material constants, in order to show why multipole theory has been successful in describing many physical phenomena despite the origin dependence of many of the expressions used to describe the material constants.

The rest of the paper is organized as follows. A brief overview of the relevant theoretical background is given in Sec. 2 and the definitions of the constitutive relations provided by multipole theory and by the transformed multipole theory by Raab and de Lange are presented and compared. In Sec.3 and Sec.4, the main results of this work are derived and discussed. It is shown that the scattering amplitude and the absorption coefficient are connected to the electric permittivity. They provide expressions for the permittivity that correspond to the definition obtained from transformed multipole theory. Our results relate the permittivity to well-known measurable quantities, such as the scattering amplitude and the absorption coef- 
ficient, providing a way to determine the permittivity experimentally, also in the optical region.

Alternative origin-independent expressions for the material constants are discussed in Sec.5, and compared to results obtained in the analysis of the scattering amplitude and absorption coefficients.

The inverse permeability is discussed in detail in Sec.6, together with the magnetizability in the microscopic case. In Sec.7, the wave equation in terms of the material constants is derived and discussed. Calculations of the origin-independent expressions of the electric permittivity and the inverse permeability are reported in Sec.8. Special attention is given to their dispersion curves. Concluding remarks and an outlook are given in Sec.9.

\section{Maxwell's equations and the constitutive relations}

Maxwell's equations form the basis of all electromagnetic phenomena, providing a complete description of the interactions between charged particles and electromagnetic fields when the particle degrees of freedom are fixed and the sources are specified. A covariant formulation of electrodynamics gives Maxwell's equations in the form (SI units) [12]

$$
\begin{aligned}
\nabla \cdot \boldsymbol{B} & =0 \\
\nabla \wedge \boldsymbol{E}+\frac{\partial \boldsymbol{B}}{\partial t} & =0 \\
\nabla \cdot \boldsymbol{D} & =\rho \\
\nabla \wedge \boldsymbol{H} & =\frac{\partial \boldsymbol{D}}{\partial t}+\boldsymbol{J}
\end{aligned}
$$

where $\boldsymbol{E}$ and $\boldsymbol{B}$ are the electric and magnetic fields, $\boldsymbol{D}$ and $\boldsymbol{H}$ the auxiliary fields and $\boldsymbol{\rho}$ and $\boldsymbol{J}$ the macroscopic free charge and current density (not including bound charges and currents), respectively.

In order to have a complete description of a macroscopic system, it is necessary to specify the relation between the electric and magnetic fields and the corresponding auxiliary fields. The auxiliary fields are thus not uniquely defined until the relation between them and the basic fields is specified through the so-called constitutive relations.

Before defining the constitutive relations, we note that in the following discussion, only non-magnetic media are considered. However, the results obtained below can be straightforwardly extended to the case of magnetic media. Furthermore, the covariant Maxwell equations eqs.(1)-(4) describe all kinds of homogeneous, dielectric, magnetic, anisotropic and chiral media [12]. In the following we will also assume that the external perturbation is described by electromagnetic plane waves of the general form

$$
\boldsymbol{F}=\boldsymbol{F}_{0} \exp [\imath(\boldsymbol{k} \cdot \boldsymbol{r}-\omega t)]
$$

where $\boldsymbol{F}$ refers to the fields $\boldsymbol{E}$ and $\boldsymbol{B}, \boldsymbol{F}_{0}$ their amplitude, $\omega$ the frequency of the oscillating fields and $\boldsymbol{k}$ the wave vector. The wave vector is related to the frequency 
of the fields and to the refractive index $n$ through the relation

$$
\boldsymbol{k}=\frac{\omega n \boldsymbol{\sigma}}{c}
$$

where $\boldsymbol{\sigma}$ defines the direction of propagation of the wave. In vacuum, the constitutive relations are

$$
\boldsymbol{D}=\varepsilon_{0} \boldsymbol{E} \quad \boldsymbol{H}=\mu_{0}^{-1} \boldsymbol{B}
$$

A general definition of the auxiliary response fields in terms of induced multipole moments, up to the electric octopole-magnetic quadrupole order, is given by [13]

$$
\begin{gathered}
D_{\imath}=\varepsilon_{0} E_{\imath}+P_{\imath}-\frac{1}{2} \nabla_{\jmath} Q_{\imath \jmath}+\frac{1}{6} \nabla_{k} \nabla_{\jmath} Q_{\imath \jmath k}-\ldots \\
H_{\imath}=\mu_{0}^{-1} B-M_{\imath}+\frac{1}{2} \nabla_{\jmath} M_{\imath \jmath}-\ldots
\end{gathered}
$$

In these equations and throughout this paper, implicit summation over repeated indices will be used (Einstein summation convention). In these equations we have also introduced the electric constant $\varepsilon_{0}$, the magnetic constant $\mu_{0}$, and $P_{i}, Q_{\imath \jmath}$, $Q_{\imath \jmath k}, M_{\imath}, M_{\imath \jmath}$ represent the macroscopically induced electric and magnetic multipole moments, respectively. The induced multipole moments can be expressed in terms of molecular responses to electric and magnetic fields (see for instance Ref. [14]). Considering an electromagnetic plane wave eq.(5), the multipole moments in eqs.(8) and (9) can be rewritten in terms of the fields. Collecting terms in the electric and magnetic fields leads to the following set of constitutive relations

$$
\begin{aligned}
& D_{\imath}=A_{\imath \jmath} E_{\jmath}+T_{\imath \jmath} B_{\jmath} \\
& H_{\imath}=U_{\imath \jmath} E_{\jmath}+X_{\imath \jmath} B_{\jmath}
\end{aligned}
$$

introducing the material constants $A_{\imath \jmath}, T_{\imath \jmath}, U_{\imath \jmath}$ and $X_{\imath \jmath}$. $A_{\imath \jmath}$ is the permittivity, $T_{\imath \jmath}$ and $U_{\imath \jmath}=T_{\imath \jmath}^{\dagger}$ the magneto-electric coefficients and $X_{\imath \jmath}$ the inverse permeability. In this definition, the permittivity appears as a purely electric property and the inverse permeability as a purely magnetic property, whereas the magneto-electric coefficients couple the electric and magnetic fields. In eqs.(10)-(11) we have truncated the expansion of the auxiliary fields in order to obtain linear constitutive relations.

The explicit expressions for the material constants in eqs.(10)-(11) provided by multipole theory, up to the electric octopole-magnetic quadrupole order and in terms 
of molecular responses, are [1]

$$
\begin{aligned}
A_{\imath \jmath} & =\varepsilon_{0} \delta_{\imath \jmath}+\alpha_{\imath \jmath}+\frac{1}{2} \imath\left(a_{\imath \jmath k}-a_{\jmath k \imath}\right) k_{k} \\
& -\frac{1}{6}\left(b_{\imath \jmath k l}+b_{\jmath \imath k l}\right) k_{k} k_{l}+\frac{1}{4} d_{\imath k \jmath l} k_{k} k_{l} \\
T_{\imath \jmath} & =-\imath G_{\imath \jmath}^{\prime}+\frac{1}{2}\left(H_{\imath \jmath k}^{\prime}-L_{\imath k \jmath}^{\prime}\right) k_{k} \\
U_{\imath \jmath} & =-\imath G_{\jmath \imath}^{\prime}-\frac{1}{2}\left(H_{\jmath \imath k}^{\prime}-L_{\jmath k \imath}^{\prime}\right) k_{k} \\
X_{\imath \jmath} & =\frac{1}{\mu_{0}} \delta_{\imath \jmath}-\chi_{\imath \jmath}
\end{aligned}
$$

The definitions, from time-dependent perturbation theory, of the various molecular response terms appearing in eqs.(12)-(15) are given in Table 1.

\begin{tabular}{|l|l|}
\hline Tensor & Definition \\
\hline$\alpha_{\imath \jmath}$ & $\frac{2}{\hbar} \sum_{s} \omega_{s n} Z_{s n} \operatorname{Re}\left\{<P_{\imath}>_{n s}<P_{\jmath}>_{s n}\right\}$ \\
$a_{\imath \jmath k}$ & $\frac{2}{\hbar} \sum_{s} \omega_{s n} Z_{s n} \operatorname{Re}\left\{<P_{\imath}>_{n s}<Q_{\jmath k}>_{s n}\right\}$ \\
$G_{\imath \jmath}^{\prime}$ & $-\frac{2}{\hbar} \sum_{s} \omega Z_{s n} \operatorname{Im}\left\{<P_{\imath}>_{n s}<M_{\jmath}>_{s n}\right\}$ \\
$b_{\imath \jmath k l}$ & $\frac{2}{\hbar} \sum_{s} \omega_{s n} Z_{s n} \operatorname{Re}\left\{<P_{\imath}>_{n s}<Q_{\jmath k l}>_{s n}\right\}$ \\
$d_{\imath \jmath k l}$ & $\frac{2}{\hbar} \sum_{s} \omega_{s n} Z_{s n} \operatorname{Re}\left\{<Q_{\imath \jmath}>_{n s}<Q_{k l}>_{s n}\right\}$ \\
$H_{\imath \jmath k}^{\prime}$ & $-\frac{2}{\hbar} \sum_{s} \omega Z_{s n} \operatorname{Im}\left\{<P_{\imath}>_{n s}<M_{\jmath k}>_{s n}\right\}$ \\
$L_{\imath \jmath k}^{\prime}$ & $-\frac{2}{\hbar} \sum_{s} \omega Z_{s n} \operatorname{Im}\left\{<Q_{\imath \jmath}>_{n s}<M_{k}>_{s n}\right\}$ \\
$\chi_{\imath \jmath}$ & $\frac{2}{\hbar} \sum_{s} \omega_{s n} Z_{s n} \operatorname{Re}\left\{<M_{\imath}>_{n s}<M_{\jmath}>_{s n}\right\}+$ \\
& $\sum_{\alpha=1}^{N} \frac{\left(q^{(\alpha)}\right)^{2}}{4 m^{(\alpha)}}\left\langle r_{\imath}^{(\alpha)} r_{\jmath}^{(\alpha)}-\left(r^{(\alpha)}\right)^{2} \delta_{\imath \jmath}\right\rangle_{n n}$ \\
\hline
\end{tabular}

Table 1. Molecular properties up to the electric-octopole magnetic-quadrupole order defining the material constants. $Z_{s n}=\left(\omega_{s n}^{2}-\omega^{2}\right)^{-1}$ and $\omega_{n s}=\frac{E_{n-E_{s}}}{\hbar}$.

It can be shown that the expressions for the material constants in eqs.(12)-(15) derived from multipole theory are origin dependent [15].

Because the material constants describe the response of a material to an electromagnetic perturbation (vide infra), they are observable quantities and thus their expressions are expected to be origin independent. As already mentioned, the auxiliary fields are however not uniquely defined by Maxwell's equations, and it is therefore necessary to introduce constitutive relations that express $\boldsymbol{D}$ and $\boldsymbol{H}$ in terms of the basic fields. There is thus a certain freedom in the definition of the constitutive relations and of the material constants, and it is possible to consider different expressions for the material constants to the ones provided by multipole theory as long as Maxwell's equations are satisfied and the wave equation is origin independent (see e.g. Ref. [1]). We will return to this point in Sec.7.

The freedom implied by Maxwell's equations should not lead to any ambiguities in the definition of the materials constants, since they are observables. Thus, the definition of the materials constants should be unique. On this basis, Raab and de Lange have proposed a transformed multipole theory of the auxiliary fields up to 
electric octopole-magnetic quadrupole order [7] that provides origin-independent expressions for the material constants. The origin independence of the material constants is imposed as a necessary requirement and, by construction, they are unique. The origin-independent expressions of the material constants resulting from this transformed multipole theory are

$$
\begin{aligned}
A_{\imath \jmath} & =\varepsilon_{0} \delta_{\imath \jmath}+\alpha_{\imath \jmath}-\frac{\imath}{\omega} \varepsilon_{\imath k l} k_{k}\left(G_{l \jmath}^{\prime}+G_{\jmath l}^{\prime}\right)+\frac{\imath}{2} k_{k}\left(a_{\imath \jmath k}-a_{\jmath \imath k}\right) \\
& +\frac{1}{2} k_{k} k_{l}\left\{\frac{2}{\omega^{2}} \varepsilon_{\imath k m} \varepsilon_{\jmath l n} \chi_{m n}-\frac{1}{\omega} \varepsilon_{\imath k m}\left(H_{\jmath m l}^{\prime}-L_{\jmath l m}^{\prime}\right)\right. \\
& \left.-\frac{1}{\omega} \varepsilon_{\jmath k m}\left(H_{\imath m l}^{\prime}-L_{\imath l m}^{\prime}\right)-\frac{1}{3}\left(b_{\imath \jmath k l}+b_{\jmath \imath k l}\right)+\frac{1}{2} d_{\imath k \jmath l}\right\} \\
T_{\imath \jmath} & =-\imath\left(G_{\imath \jmath}^{\prime}-\frac{1}{2} \omega \varepsilon_{\jmath k l} a_{k l \imath}\right)-\frac{1}{2 \omega} \varepsilon_{\imath k l} k_{k} X_{l \jmath} \\
U_{\imath \jmath} & =-\imath\left(G_{\jmath \imath}^{\prime}-\frac{1}{2} \omega \varepsilon_{\imath k l} a_{k l \jmath}\right)+\frac{1}{2 \omega} \varepsilon_{\jmath k l} k_{k} X_{\imath l} \\
X_{\imath \jmath} & =\frac{1}{\mu_{0}} \delta_{\imath \jmath}-\chi_{\imath \jmath}+\frac{1}{2} \omega\left\{\varepsilon_{\imath k l}\left(H_{\jmath k l}^{\prime}+L_{\jmath k l}^{\prime}\right)\right. \\
& +\varepsilon_{\jmath k l}\left(H_{\imath k l}^{\prime}+L_{\imath k l}^{\prime}\right)-\omega\left[\frac{1}{3}\left(b_{\imath \jmath k k}+b_{\jmath \imath k k}\right)-\frac{1}{2} d_{\imath k \jmath k}\right] \\
& \left.-\delta_{\imath \jmath}\left[\varepsilon_{k l m} H_{k l m}^{\prime}-\omega\left(\frac{1}{3} b_{k k l l}-\frac{1}{4} d_{k l k l}\right)\right]\right\}
\end{aligned}
$$

In conclusion, conventional multipole theory provides origin-dependent expressions for the material constants (see eqs.(12)-(15)), whereas applying transformed multipole theory to the auxiliary fields leads to origin-independent expressions (eqs.(16)-(19))), that are obtained by considering terms up to the electric octupolemagnetic quadrupole order.

\section{3. $\quad$ Scattering amplitude and permittivity}

In this section, we will present an alternative derivation of the expression for the permittivity that is more straightforward than transformation theory and which is based on the standard quantum-mechanical theory of the interaction between electromagnetic fields and matter.

We will demonstrate that the permittivity in eq.(16) can be obtained starting from the definition of the off-resonance, single-photon scattering amplitude. The derivation builds on the work of Healy [10], who presented a generalization of the Kramers-Heisenberg dispersion formula [16]. Healy showed that the expression for the scattering amplitude derived using perturbation theory to second order, with the minimal-coupling interaction Hamiltonian as perturbation, is identical to the expression for the scattering amplitude provided by the multipolar formulation of molecular quantum electrodynamics, where a multipolar Hamiltonian is used. However, no multipolar approximation is made, instead exact integral expressions from the multipolar formulation of molecular quantum electrodynamics are used, retaining terms to second order in the wave vector.

In order to derive an expression for the scattering amplitude in terms of induced electric and magnetic moments, we consider a Taylor expansion of the electromagnetic wave, to second order.

Let us start by considering the measurable quantity $d \sigma_{2 \leftarrow 1}$, the probability that 
a photon with wave vector $\boldsymbol{k}_{1}$ and polarization $\lambda_{1}$ is scattered by the molecule, initially in the state $\mathrm{n}(1)$ with energy $E_{1}$, into a photon with wave vector $\mathbf{k}_{\mathbf{2}}$ and polarization $\lambda_{2}$, leaving the molecule in the state $\mathrm{n}(2)$ with energy $E_{2}$. This probability is measured by the differential cross section per unit solid angle about $\boldsymbol{k}_{\mathbf{2}}$

$$
\frac{d \sigma_{2 \leftarrow 1}}{d \Omega_{2}}=\left|f_{2 \leftarrow 1}\right|^{2}=\left|-(2 \pi)^{2} k_{2} T_{2 \leftarrow 1}\right|^{2}
$$

where $f_{2 \leftarrow 1}$ is the single-photon scattering amplitude defined in terms of the transition matrix element for the process $T_{2 \leftarrow 1}[10]$ :

$$
\begin{aligned}
T_{2 \leftarrow 1}= & \left\langle k_{2} \lambda_{2}, n(2)\left|H_{\text {int }}\right| n(1), k_{1} \lambda_{1}\right\rangle+ \\
& \sum_{I} \frac{\left\langle k_{2} \lambda_{2} n(2)\left|H_{\text {int }}\right| I\right\rangle\left\langle I\left|H_{\text {int }}\right| k_{1} \lambda_{1} n(1)\right\rangle}{E_{\text {initial }}-E_{I}}
\end{aligned}
$$

The expression for the scattering amplitude derived using perturbation theory and the minimal coupling Hamiltonian for an atom interacting with electromagnetic fields is given by [10]

$$
\begin{aligned}
f_{2 \leftarrow 1} & =-k_{1}^{-1 / 2} k_{2}^{1 / 2}\left\{e_{\imath}^{\left(\lambda_{1}\right)} e_{\imath}^{\left(\lambda_{2}\right) *} \frac{e^{2}}{m}\left\langle n_{2}\left|e^{\imath\left(\boldsymbol{k}_{1}-\boldsymbol{k}_{2}\right) \cdot \boldsymbol{r}}\right| n_{1}\right\rangle\right. \\
& +\sum_{n}\left[\frac{e_{\jmath}^{\left(\lambda_{2}\right) *}(e / m)\left\langle n_{2}\left|p_{\jmath} e^{-\imath \boldsymbol{k}_{2} \cdot \boldsymbol{r}}\right| n\right\rangle e_{\imath}^{\left(\lambda_{1}\right)}(e / m)\left\langle n\left|p_{\imath} e^{-\imath \boldsymbol{k}_{1} \cdot \boldsymbol{r}}\right| n_{1}\right\rangle}{E_{1}-E_{n n_{1}}}\right. \\
& \left.\left.-\frac{e_{\imath}^{\left(\lambda_{1}\right)}(e / m)\left\langle n_{2}\left|p_{\imath} e^{\imath \boldsymbol{k}_{1} \cdot \boldsymbol{r}}\right| n\right\rangle e_{\jmath}^{\left(\lambda_{2}\right) *}(e / m)\left\langle n\left|p_{\jmath} e^{-\imath \boldsymbol{k}_{2} \cdot \boldsymbol{r}}\right| n_{1}\right\rangle}{E_{2}+E_{n n_{1}}}\right]\right\}
\end{aligned}
$$

where $e_{2}^{(\lambda)}$ is the polarization vector with polarization $\lambda$, and $E_{n_{2} n_{1}}=E_{n_{2}}-E_{n_{1}}$. Note that the hypothesis of energy conservation and off-resonance frequency has been imposed, that is $k_{1}+k_{n(1)}=k_{2}+k_{n(2)}$, where $k_{n(i)}=\frac{E_{n(i)}}{\hbar c}$.

Assuming that the wavelength of the radiation is much greater than the molecular dimension $(\boldsymbol{k} \cdot \boldsymbol{r} \ll 1)$, we can perform a Taylor expansion to second order of the exponentials

$$
\exp (-\imath \boldsymbol{k} \cdot \boldsymbol{r})=1-\imath \boldsymbol{k} \cdot \boldsymbol{r}-\frac{1}{2}(\boldsymbol{k} \cdot \boldsymbol{r})^{2}-\ldots
$$

and inserting this expansion into eq.(22), we obtain a linear combination of terms at different order in the wave vector $\boldsymbol{k}$, containing transition moments in both velocity and length gauge. Retaining terms up to second order in the Taylor expansion means including contributions of electric octopole-magnetic quadrupole order. We use the canonical commutation relations $p_{\imath}=-\frac{\imath m}{\hbar}\left[r_{\imath}, H\right]$ to express everything in the length gauge. By using the definition of a response function

$$
\langle\langle A ; B\rangle\rangle_{\omega}=-\frac{1}{\hbar} \sum_{n \neq 0}\left[\frac{\langle 0|A| n\rangle\langle n|B| 0\rangle}{\omega_{n o}-\omega}+\frac{\langle 0|B| n\rangle\langle n|A| 0\rangle}{\omega_{n o}+\omega}\right]=\langle\langle B ; A\rangle\rangle_{-\omega}
$$


and imposing energy conservation, we obtain

$$
\begin{aligned}
& f_{2 \leftarrow 1}=e_{\imath}^{\left(\lambda_{1}\right)} e_{\jmath}^{\left(\lambda_{2}\right) *}\left[\alpha_{\imath \jmath}+\frac{1}{2} \imath k_{k}\left(a_{\imath \jmath k}-a_{\jmath \imath k}\right)-\frac{1}{6} k_{k} k_{l}\left(b_{\imath \jmath k l}+b_{\jmath \imath k l}\right)\right. \\
& +\frac{1}{4} d_{\imath k \jmath l}-\imath \varepsilon_{\imath k l} k_{k}\left(G_{l \jmath}^{\prime}+G_{\jmath l}^{\prime}\right)+\varepsilon_{\imath k m} \varepsilon_{\jmath l n} k_{k} k_{l} \chi_{m n} \\
& \left.-\frac{1}{2} \varepsilon_{l k m} k_{k} k_{l}\left(H_{\jmath m l}^{\prime}-L_{\jmath l m}^{\prime}\right)-\frac{1}{2} \varepsilon_{\jmath k m} k_{k} k_{l}\left(H_{\imath m l}^{\prime}-L_{\imath l m}^{\prime}\right)\right] \\
& =e_{\imath}^{\left(\lambda_{1}\right)} e_{\jmath}^{\left(\lambda_{2}\right) *} A_{\imath \jmath}
\end{aligned}
$$

where the definition of $A_{i j}$ introduced in eq.(16) has been used. Eq.(25) defines the scattering amplitude in terms of the electric permittivity invoking the originindependent definition of this material constant provided by Raab and De Lange in their transformed multipole theory eq.(16). In the appendix, we present an alternative derivation of this result using the expression for the scattering amplitude provided by a multipolar formulation of molecular quantum electrodynamics.

In this derivation we have retained terms at the second order in both: (1) the expansion of the external perturbation (eq.(23)), and (2) the vector potential in the Hamiltonian (eq.(21)). The expression for the oscillator strength is then the square of eq.(25). Comparing this expression to the one derived by Bernadotte et al.[17], we can see that they apparently differ. This is due to the fact that Bernadotte et al. do not include the quadratic term of the vector potential in the Hamiltonian, thus obtaining an expression containing only the leading-order contribution. A result more directly comparable to the one of Bernadotte et al. will be derived and discussed in the next section, where the quadratic term of the vector potential in the Hamiltonian will be neglected.

The derivation of the permittivity presented here provides an indirect route for measuring the permittivity, since it has been shown to be related to a well-known measurable quantity, the differential cross section, expressable as a linear combination of polarizabilities. We have also indirectly confirmed the validity of the expression derived by Raab and De Lange in transformed multipole theory.

\section{Absorption coefficients and permittivity}

In the previous section, an origin-independent expression for the permittivity was derived from the quantum-mechanical expression for the differential cross section, providing a unique definition of this material constant. It was also shown that the expression obtained for the permittivity is equivalent to the expression provided by transformed multipole theory. Here, we show that an equivalent result can be achieved from the definition of the absorption coefficient in terms of the response function of the current density. This derivation is similar to the former approach, but allows us to also consider the current density.

Let us consider again a photon with wave vector $\boldsymbol{k}_{1}$ and polarization $\lambda_{1}$, that is scattered by a molecule of volume $\mathrm{V}$ and linear dimension $\mathrm{L}$, initially in the state $\mathrm{n}(1)$ with energy $E_{1}$, into a photon with wave vector $\mathbf{k}_{2}$ and polarization $\lambda_{2}$, leaving the molecule in the state $\mathrm{n}(2)$ with energy $E_{2}$. The probability that a photon would be detected with polarization $\lambda_{2}$ and wave vector $k_{2}$ is the square of the amplitude: 


$$
\begin{array}{r}
T_{2 \leftarrow 1}=\exp (-\imath k L)\left[\delta_{\lambda_{1} \lambda_{2}} \delta_{k_{1} k_{2}}-\left(\frac{2 \pi \imath L}{k c^{2} V}\right)\left\{\frac{e}{m} q\left(k_{2}-k_{1}\right) n_{\lambda_{2}}\left(k_{2}\right) \cdot n_{\lambda_{1}}\left(k_{1}\right)\right.\right. \\
\left.\left.+\ll j_{\lambda_{2}}\left(k_{2}\right) ; j_{\lambda_{1}}\left(k_{1}\right) \gg_{k c}\right\}\right]
\end{array}
$$

expressed in terms of the current density [11]. Retaining terms linear in L we get

$$
\left|T_{2 \leftarrow 1}\right|^{2}=1+\left(\frac{4 \pi}{\omega c V}\right) \Im \ll j_{\lambda_{1}}\left(k_{1}\right) ; j_{\lambda_{1}}\left(k_{1}\right) \gg_{\omega}
$$

The absorption coefficient describing the decrease in intensity per unit length of matter in the ground state is

$$
a=-\left(\frac{4 \pi}{\omega c V}\right) \Im \ll j_{\lambda_{1}}\left(k_{1}\right) ; j_{\lambda_{1}}\left(k_{1}\right) \gg_{\omega}
$$

where $\omega=k c$.

From a quantization of the fields and the Hamiltonian describing the molecular system, the current density can be expressed as:

$$
j_{\lambda}(\boldsymbol{k})=\boldsymbol{n}_{\lambda}(\boldsymbol{k}) \cdot \int d \boldsymbol{r} \boldsymbol{j}(\boldsymbol{r}) \exp (-\imath \boldsymbol{k} \cdot \boldsymbol{r})
$$

where the current density operator $\boldsymbol{j}(\boldsymbol{r})$ is

$$
\begin{aligned}
\boldsymbol{j}(\boldsymbol{r})= & \sum_{\text {spin }}\left[\frac{e}{2 m}\left(\imath \boldsymbol{\nabla} \psi^{\dagger}(\xi)\right) \psi(\xi)-\imath \psi^{\dagger}(\xi) \boldsymbol{\nabla} \psi(\xi)\right. \\
& \left.-\frac{2 e}{c} \psi^{\dagger}(\xi) \psi(\xi) \boldsymbol{A}(\boldsymbol{r})+\boldsymbol{\nabla} \wedge\left(\psi^{\dagger}(\xi) \boldsymbol{\sigma} \psi(\xi)\right)\right]
\end{aligned}
$$

$\psi(\xi)$ is here the electron field operator, satisfying the wave equation, and $\boldsymbol{\sigma}$ the Pauli spin operators. We exclude from the current density operator the field-dependent term and the spin term - that is, we consider low-intensity light sources and closedshell systems only. Inserting the definition of the current density operator eq.(30) into eq.(29), we obtain

$$
\left.j_{\lambda}(\boldsymbol{k})=\boldsymbol{n}_{\lambda}(\boldsymbol{k}) \cdot \int d \boldsymbol{r} \frac{e}{2 m}\left(\imath \boldsymbol{\nabla} \psi^{\dagger}(\xi)\right) \psi(\xi)-\imath \psi^{\dagger}(\xi) \boldsymbol{\nabla} \psi(\xi)\right) \exp (-\imath \boldsymbol{k} \cdot \boldsymbol{r})
$$

Inserting the Taylor expansion to second order of the exponential given in eq.(23) in the integral in eq.(31) and considering the component of $\boldsymbol{r}$ along $\boldsymbol{k}$ and $\boldsymbol{\nabla}$ along $\boldsymbol{n}_{\lambda}$, eq.(31) takes the form

$$
\begin{aligned}
j_{\lambda}(\boldsymbol{k})= & \frac{e}{m}\left\{\int d \xi \psi^{\dagger}(\xi)\left(-\imath \nabla_{\lambda}\right) \psi(\xi)-\int d \xi \psi^{\dagger}(\xi) \imath k_{l} r_{l}\left(-\imath \nabla_{\lambda}\right) \psi(\xi)\right. \\
& \left.-\frac{1}{2} \int d \xi \psi^{\dagger}(\xi) k_{l} k_{m} r_{l} r_{m}\left(-\imath \nabla_{\lambda}\right) \psi(\xi)\right\}
\end{aligned}
$$


Using the identities

$$
\begin{aligned}
r_{l}\left(-\imath \nabla_{\lambda}\right) & = \pm \frac{1}{2 \hbar} l_{k}+\frac{1}{2}\left[r_{l}\left(-\imath \nabla_{\lambda}\right)+r_{\lambda}\left(-\imath \nabla_{l}\right)\right] \\
r_{l} r_{m}\left(-\imath \nabla_{\lambda}\right) & =\frac{1}{3}\left[r_{l} r_{m}\left(-\imath \nabla_{\lambda}\right)+r_{l}\left(-\imath \nabla_{m}\right) r_{\lambda}+\left(-\imath \nabla_{l}\right) r_{m} r_{\lambda}\right] \\
& +\frac{1}{3 \hbar}\left[r_{l} l_{\lambda}+l_{\lambda} r_{l}\right]
\end{aligned}
$$

we can rewrite the response function of the current density in terms of magnetic and electric moments. Note that the indices $l$ and $m$ are contracted with the wave vector, $l_{k}$ is the angular momentum and an explicit choice for the orientation of the axis has been made. Defining the magnetic dipole and quadrupole moments as: $m_{\imath}=\frac{e}{2 m} l_{\imath}, m_{\imath \jmath}=\frac{e}{3 m}\left(r_{\imath} l_{\imath}+l_{\imath} r_{\jmath}\right)$ and using the Levi-Civita tensor, the current density for an arbitrary direction $\lambda$ is

$$
\begin{aligned}
j_{\lambda}(\boldsymbol{k}) & =\frac{e}{\hbar} p_{\lambda}-\frac{\imath}{\hbar} k_{l} \varepsilon_{k l \lambda} m_{k}-\frac{\imath e}{2 m \hbar} k_{l}\left(r_{l} p_{\lambda}+r_{\lambda} p_{l}\right) \\
& -\frac{1}{2 \hbar} k_{l} k_{m} \varepsilon_{k l \lambda} m_{k m}-\frac{e}{6 m \hbar} k_{l} k_{m}\left(r_{l} r_{m} p_{\lambda}+r_{l} p_{m} r_{\lambda}+p_{l} r_{m} r_{\lambda}\right)
\end{aligned}
$$

where $p_{\imath}$ is a component of the linear momentum operator. Eq.(35) has been obtained using simple algebraic manipulations, canonical commutation relations and the identities

$$
\begin{aligned}
p_{\lambda} & =-\frac{\imath m}{\hbar}\left[r_{\lambda}, H\right] \\
\langle\langle[A, H] ; B\rangle\rangle & =\hbar \omega\langle\langle A ; B\rangle\rangle-\langle[A, B]\rangle \\
\langle\langle A ;[B, H]\rangle\rangle & =\hbar \omega\langle\langle A ; B\rangle\rangle+\langle[A, B]\rangle
\end{aligned}
$$

The response functions that appear in the definition of the absorption coefficient, eq. (28) can be calculated in a similar manner. Note that the complex conjugate of eq. (35) should be considered for an arbitrary orthogonal direction $\lambda^{\prime}$. Thus, for two arbitrary directions $\lambda=\imath$ and $\lambda^{\prime}=\jmath$, the response function of the current density is

$$
\begin{aligned}
\ll j_{\imath}(\boldsymbol{k}) ; j_{\jmath}^{\dagger}(\boldsymbol{k}) \gg_{\omega} & =\frac{\omega^{2}}{\hbar^{2}}\left\{\frac{1}{m} \delta_{\imath \jmath}+\alpha_{\imath \jmath}-\frac{\imath}{\omega}\left(\varepsilon_{\imath k l} k_{k}\left(G_{l \jmath}^{\prime}+G_{\jmath l}^{\prime}\right)\right.\right. \\
& +\frac{\imath}{2}\left(k_{k}\left(a_{\imath \jmath k}-a_{\jmath \imath k}\right)-\frac{1}{6} k_{k} k_{l}\left(b_{\imath \jmath k l}+b_{\jmath \imath k l}\right)\right. \\
& +\frac{1}{4} d_{\imath k \jmath l}-\frac{1}{2 \omega} k_{k} k_{l} \varepsilon_{\imath k m}\left(H_{\jmath m l}^{\prime}-L_{\jmath l m}^{\prime}\right) \\
& -\frac{1}{2 \omega} k_{k} k_{l} \varepsilon_{\jmath k m}\left(H_{\imath m l}^{\prime}-L_{\imath l m}^{\prime}\right) \\
& \left.+\frac{1}{\omega^{2}} k_{k} k_{l} \varepsilon_{\imath k m} \varepsilon_{\jmath l n} \chi_{m n}\right\}
\end{aligned}
$$

Comparing eq.(39) with the expression for the permittivity obtained by Raab and de Lange eq.(16), keeping terms up to electric octopole-magnetic quadrupole or- 
der [7], one can see that these expressions are identical. Thus, an origin-independent expression of the permittivity can be obtained from the response function of the current density. This result is equivalent to the one derived by Bernadotte et al. [17].

It is important to note that our calculation have started with quantities that are origin independent because they are physical/observable quantities, namely the external electromagnetic field and the current density. As a consequence, the expressions derived are also origin independent at each order in the Taylor expansion. Retaining only first-order terms in the expansion would lead to an expression for the current density proportional to the polarizability $\left(\alpha_{\imath \jmath}\right)$ [11], which is well-known to be origin independent. In our derivation, we have not imposed any constraints nor applied any transformation theory in order to derive an origin-independent expression for the permittivity as done in earlier work [7, 8]. Instead, standard quantummechanical theory for the interaction between matter and electromagnetic fields is used.

In accordance with the discussion above, we can define the absorption coefficient in terms of the permittivity $A_{\lambda_{1} \lambda_{1}}$ as

$$
a=-\left(\frac{4 \pi \omega}{\hbar^{2} c V}\right) \Im\left(A_{\lambda_{1} \lambda_{1}}\right)
$$

Thus, a direct link between the electric permittivity and a physical observable quantity, the absorption coefficient, has been provided.

\section{Covariant formulation of the constitutive relations}

Exploiting the non-uniqueness of the auxiliary fields (discussed in section 2), alternate definitions for the auxiliary fields and the material constants can be obtained within the relativistic covariant theory of electromagnetism [12]. Referring to the discussion in Refs. [3, 9, 12], the constitutive relations can be written

$$
\begin{aligned}
& D_{\imath}^{\prime}=A_{\imath \jmath} E_{\jmath}+T_{\imath \jmath} B_{\jmath} \\
& H_{\imath}^{\prime}=U_{\imath \jmath} E_{\jmath}+X_{\imath \jmath} B_{\jmath}
\end{aligned}
$$

where $D_{\imath}^{\prime}$ and $H_{\imath}^{\prime}$ are the auxiliary fields in covariant form that satisfy Maxwell's equations (free of sources)

$$
\begin{aligned}
\nabla \cdot \boldsymbol{D}^{\prime} & =0 \\
\nabla \wedge \boldsymbol{H}^{\prime} & =\frac{\partial \boldsymbol{D}}{\partial t}
\end{aligned}
$$

The material constants in eqs. (41) and (42), to electric octopole-magnetic quadrupole order, are defined as [9] 


$$
\begin{aligned}
A_{\imath \jmath} & =\varepsilon_{0} \delta_{\imath \jmath}+F_{\imath \jmath}-\frac{1}{12} k_{k} k_{l} W_{\imath \jmath k l} \\
T_{\imath \jmath} & =-\imath t_{\imath \jmath}^{\prime}+k_{k} h_{\imath \jmath k}^{\prime} \\
U_{\imath \jmath} & =-\imath t_{\jmath \jmath}^{\prime}-k_{k} h_{\jmath \imath k}^{\prime} \\
X_{\imath \jmath} & =-\frac{1}{\mu_{0}} \delta_{\imath \jmath}-m_{\imath \jmath}
\end{aligned}
$$

where [9]

$$
\begin{aligned}
F_{\imath \jmath} & =\alpha_{\imath \jmath} \\
t_{\imath \jmath}^{\prime} & =G_{\imath \jmath}^{\prime}-\frac{1}{2} \omega \varepsilon_{\jmath k l} a_{k l \imath} \\
W_{\imath \jmath k l} & =\left(b_{\imath \jmath k l}+b_{\jmath \imath k l}+b_{k \imath \jmath l}+b_{l \jmath \imath k}\right)-\left(d_{\imath \jmath k l}+d_{\imath k \jmath l}+d_{\jmath k \imath l}\right) \\
h_{\imath \jmath k}^{\prime} & =\frac{1}{4}\left(H_{\jmath \imath k}^{\prime}+H_{k \imath \jmath}^{\prime}-2 L_{\jmath k \imath}^{\prime}\right)-\frac{1}{16} \delta_{\jmath \imath}\left(H_{k l l}^{\prime}+H_{l l k}^{\prime}-2 L_{k l l}^{\prime}\right) \\
& -\frac{1}{16} \delta_{\imath k}\left(H_{\jmath l l}^{\prime}+H_{l l \jmath}^{\prime}-2 L_{\jmath l l}^{\prime}\right)+\frac{1}{12} \omega \varepsilon_{\imath l m} b_{l \jmath k m} \\
m_{\imath \jmath} & =\chi_{\imath \jmath}-\frac{1}{4} \omega\left(\varepsilon_{\imath k l} H_{k \jmath l}^{\prime}+\varepsilon_{\jmath k l} H_{k \imath l}^{\prime}\right)-\frac{1}{48} \omega^{2}\left(\varepsilon_{\imath k l} \varepsilon_{\jmath m n}+\varepsilon_{\jmath k l} \varepsilon_{\imath m n}\right) d_{k m n l}
\end{aligned}
$$

The tensors which appear in these expressions are given in Table 1 . Note that eqs. (49)-(53) are origin independent, and thus also the material constants in eqs. (45)-(48).

Using the tensors in eqs. (49)-(53), we can rewrite the expressions for the permittivity and permeability given in the previous section eqs. (16)-(19) as

$$
\begin{aligned}
A_{\imath \jmath} & =\varepsilon_{0} \delta_{\imath \jmath}+F_{\imath \jmath}+k_{k} k_{l}\left(\frac{1}{\omega^{2}} \varepsilon_{\imath k m} \varepsilon_{\jmath l n} m_{m n}-\frac{1}{\omega} \varepsilon_{\imath k m} h_{\jmath m l}^{\prime}\right. \\
& \left.-\frac{1}{\omega} \varepsilon_{\jmath l n} h_{\imath n k}^{\prime}-\frac{1}{12} W_{\imath \jmath k l}\right) \\
X_{\imath \jmath} & =-\frac{1}{\mu_{0}} \delta_{\imath \jmath}-m_{\imath \jmath}+\omega \varepsilon_{\imath k l} h_{\jmath k l}^{\prime}+\omega \varepsilon_{\jmath k l} h_{\imath l k}^{\prime} \\
& +\left(2 \delta_{\imath k} \delta_{\jmath l}-\delta_{\imath \jmath} \delta_{k l}\right) \frac{\omega^{2}}{24} W_{k l m m}
\end{aligned}
$$

Eqs.(54) and (55) clearly differ from eqs.(45) and (48). Two different covariant and origin-independent definitions of the material constants are thus achieved.

It is clear that the material constants cannot be uniquely defined. In order to discriminate between the different expressions for the material constants, and compare them with experimental results, it is necessary to add more information to the theory.

The analysis presented in Secs.3 and 4, which is a first principles approach to the problem, provides a definition of the permittivity and of the material constants by transformed multipole theory, that are origin independent at all orders in the multipole expansion, and that can be related to experimentally observable quantities. 


\section{Inverse permeability and magnetizability}

An origin-independent expression for the inverse permeability has been derived by Raab and de Lange using a transformed multipole theory for the auxiliary fields [7], see eq.(19) (or from the covariant formulation in eq.(55)). In the microscopic case, an origin-independent expression for the frequency-dependent magnetizability, equivalent to eq.(19), has also been derived by the same authors [8]

$$
\begin{aligned}
\alpha_{\imath \jmath}^{m} & =\chi_{\imath \jmath}-\frac{1}{2} \omega\left\{\varepsilon_{\imath k l}\left(H_{\jmath k l}^{\prime}+L_{\jmath k l}^{\prime}\right)\right. \\
& +\varepsilon_{\jmath k l}\left(H_{\imath k l}^{\prime}+L_{\imath k l}^{\prime}\right)-\omega\left[\frac{1}{3}\left(b_{\imath \jmath k k}+b_{\jmath \imath k k}\right)-\frac{1}{2} d_{\imath k \jmath k}\right] \\
& \left.-\delta_{\imath \jmath}\left[\varepsilon_{k l m} H_{k l m}^{\prime}-\omega\left(\frac{1}{3} b_{k k l l}-\frac{1}{4} d_{k l k l}\right)\right]\right\}
\end{aligned}
$$

where $\alpha_{\imath \jmath}^{m}$ indicates the second-order magnetic response (frequency-dependent magnetizability), which is different from the definition of the magnetizability provided by multipole theory to lowest order $\chi_{\imath \jmath}$, to which $\alpha_{\imath \jmath}^{m}$ reduces in the static limit.

The procedure used to derive eq.(56) in Ref. [8] relies on a number of assumptions that the expression for the magnetizability is supposed to fulfil. The expression in eq.(56) matches the definition of the inverse permeability given in eq.(19). Although we cannot isolate a simple linear expression for the inverse permeability from the constitutive relations and the expressions for the material constants, the correspondence between eq.(56) and eq.(19) gives a strong support for this form of the inverse permeability. However, with the exception of this indirect argument, there is no physical reason not to assume eq.(48) as an equally good definition of the inverse permeability, since it is covariant, origin independent and satisfies the Maxwell equations.

It is important to note that, according to transformed multipole theory, in order to derive an origin-independent definition of the material constants, it is necessary to include contributions up to the electric octopole-magnetic quadrupole order (see ref.[7]). This confirms that when going beyond the dipole approximation in the definition of the permittivity, as derived from the absorption coefficient (or the scattering amplitude), contributions up to the electric octopole-magnetic quadrupole order must be included.

Eq.(16) can thus be considered the proper definition of the electric permittivity, whereas the definition of the inverse permeability needs further investigations. Neverthless, considering the intimate relation between the electric permittivity and the inverse permeability, our results for the permittivity suggests that the proper expression for the inverse permeability is provided by eq.(19).

\section{Wave equation}

The starting point for deriving the wave equation is the Maxwell equations for the auxiliary fields and the constitutive relations given in eqs.(3)-(4) and eqs.(10)(11), respectively. Taking an electromagnetic plane wave (eq.(5)) and inserting the definitions of the auxiliary fields in terms of the material constants in the Maxwell equations, the two wave equations 


$$
\begin{aligned}
& \left(\frac{1}{\omega} \varepsilon_{\imath p q} k_{p} U_{q \jmath}+A_{\imath \jmath}+\frac{1}{\omega^{2}} \varepsilon_{\imath p q} \varepsilon_{l k \jmath} k_{k} k_{p} X_{q l}+\frac{1}{\omega} \varepsilon_{l k \jmath} k_{k} T_{\imath l}\right) E_{0 \jmath}=0 \\
& \left(\frac{1}{\omega} \varepsilon_{\imath p q} k_{p} X_{q \jmath}+T_{\imath \jmath}-\frac{c^{2}}{\omega^{2}} \varepsilon_{\imath p q} \varepsilon_{l k \jmath} k_{k} k_{p} U_{q l}-\frac{c^{2}}{\omega} \varepsilon_{l k \jmath} k_{k} A_{\imath l}\right) B_{0 \jmath}=0
\end{aligned}
$$

are derived, where Faraday's law $\nabla \wedge \boldsymbol{E}=-\frac{\partial \boldsymbol{B}}{\partial t}$ is used to express $\boldsymbol{B}$ in terms of $\boldsymbol{E}$ in the first wave equation, and $\boldsymbol{E}$ in terms of $\boldsymbol{B}$ in the second.

The wave equations eqs.(57) and (58) are origin independent and do not depend on the form of the material constants. This provides a degree of freedom in the definition of the material constants, subject to the constraint that the wave equation remains origin independent. By substituting the two different definitions of the material constants given in eqs.(16)-(19) and (45)-(48), it can be shown that they both lead to the same wave equation.

Note that the definitions of the material constants given by multipole theory, eqs.(12)-(15), also lead to the same origin-independent wave equation. This is somewhat surprising since the expressions for the material constants provided by multipole theory are origin dependent. However, this explains why it has been a successful theory to explain several observable phenomena, although it gives unphysical results for reflection effects. In the latter case, it is necessary to consider the transformed material constants in order to have origin-independent expressions[1]. Reflection effects can also be used to experimentally to help elucidate which of the two originindependent definitions for the material constants introduced above correspond to experimental interactions. The study of the refractive index of chiral molecules, such as helicene, could also provide valuable insights. In fact, the refractive index of chiral molecules is defined in terms of the four material constants[18], appearing at the same order of magnitude, so that the referactive index values would be different for the different definitions of the material constants.

In contrast, solving the wave equation in terms of the fields and of the material constants leads to a good description of various optical effects, but does not provide any constraints for deciding on the proper definition of the material constants.

\section{Numerical investigations}

We have in the previous sections discussed the two different origin-independent expressions for the electric permittivity and inverse permeability as presented by Raab and de Lange in eqs.(16)-(19) and eqs.(45)-(48). We have shown that the electric permittivity obtained from transformed multipole theory can be derived from experimentally observable quantities, both the scattering amplitude and the absorption cross section. Albeit indirectly, this gives support for the expression for the inverse permeability as obtained from the transformed multipole theory.

We now turn our attention to a numerical investigation of the effects of the two different forms of the permittivity and inverse permeability in order to explore the numerical differences between the two expressions, and thus in which situations we can expect to be able to distinguish between the two expressions through experimental observations. 


\subsection{Computational details}

All calculations presented in this work have been performed using a development version of the Dalton program package [19]. Our calculations have been performed using density functional theory with the B3LYP functional [20] together with the augmented correlation-consistent double-zeta basis (aug-cc-pVDZ) of Dunning and Woon [21, 22]. The inverse permeability has been calculated in the presence of a dynamic external perturbation (electromagnetic waves) for different frequencies of the applied external electromagnetic field.

We have chosen the benzene molecule as a test system because it is comparatevely large, elctronic rich and symmetric. Therefore a strong response can be expected, highlighting interesting aspects of the dispersion curve of the inverse permeability. The molecule has been placed in the yz-plane with the center of mass at the origin of the coordinates system and with the long axis of the molecule along the $\mathrm{z}$ axis. Due to the strong origin dependence observed for the conventional static magnetizability [23] when using a finite basis set, we have used London atomic orbitals (LAOs) [24] in the calculation of the conventional diamagnetic and paramagnetic contributions to the magnetizability $[25,26]$. The gauge-origin independence of the two expressions for the inverse permeability as well as of the electric permittivity has been verified by shifting the gauge origin. The molecular responses that contribute to the electric permittivity and inverse permeability have been implemented in the Dalton program as straightforward extensions to the complex polarization propagator approach. [27]

The two lowest excitation energies of the benzene molecule are at this computational level $\omega=0.198893 \mathrm{E}_{h}$ and $\omega=0.221012 \mathrm{E}_{h}$, corresponding to states of $B_{2 u}$, $B_{1 g}$ symmetry, respectively. We note that the first of these states is electric dipole allowed and electric quadrupole (and thus magnetic dipole) forbidden, whereas the second excited state is electric quadrupole allowed and electric dipole forbidden.

\subsection{Inverse permeability}

The two different definitions of the inverse permeability have been calculated considering the origin-independent definitions given in eqs.(19) and (48), respectively. A range of frequencies between $\omega=0 \mathrm{E}_{h}$ and $\omega=0.26 \mathrm{E}_{h}$ has been used, covering the first five excitation energies, in order to investigate the behaviour of the inverse permeability close to resonances.

In order to check the origin dependence of the expressions for the inverse permeability, we have first located the center of mass of the benzene molecule at the origin of the coordinate system, and then we have shifted the gauge origin and the dipole origin by 1 Bohr. The use of LAOs ensure gauge-origin independence for tensors that depend on the magnetic dipole operator, but not for the tensor $H_{\imath \jmath k}$ which include the magnetic quadrupole moment for which an implementation using the London orbital approach does not yet exist. The error due to this fact is fairly small, as can be seen from Tables 2 and 3 .

In Tables 2 and 3 we report the results of our calculations for the inverse permeability. In this work, both the expression provided by transformed multipole theory and by the covariant formulation are calculated. Calculations for the xx-component and zz-component of the tensor $X_{\imath \jmath}\left(X_{x x}, X_{z z}\right)$, at the origin and when a shift along the y-axis, are performed. Due to the symmetry of the benzene molecule, analogous

results to $X_{z z}$ have been obtained for the $X_{y y}$ component. Shifts along the $\mathrm{x}$ or the $\mathrm{z}$ axis show the same behaviour. In the calculation, a damping factor of 0.005 a.u. has been used. We will in the following refer to eq.(19) as the "Raab and de Lange expression" (RL) and to eq.(48) as the "alternative expression" (A). 


\begin{tabular}{|lccll|}
\hline$\omega\left(\mathrm{E}_{h}\right)$ & $X_{x x}^{R L}\left(D_{y}=0\right)$ & $X_{x x}^{R L}\left(D_{y}=1\right)$ & $X_{x x}^{A}\left(D_{y}=0\right)$ & $X_{x x}^{A}\left(D_{y}=1\right)$ \\
\hline 0,001 & $-20,555$ & $-20,557$ & $-20,507$ & $-20,509$ \\
0,100 & $-26,097$ & $-26,091$ & $-20,640$ & $-20,631$ \\
0,150 & $-36,217$ & $-36,199$ & $-21,180$ & $-21,154$ \\
0,170 & $-43,896$ & $-43,870$ & $-21,803$ & $-21,767$ \\
0,180 & $-49,381$ & $-49,349$ & $-22,330$ & $-22,288$ \\
0,190 & $-56,763$ & $-56,726$ & $-23,111$ & $-23,061$ \\
0,200 & $-67,475$ & $-67,431$ & $-24,303$ & $-24,243$ \\
0,205 & $-75,060$ & $-75,011$ & $-25,140$ & $-25,074$ \\
0,210 & $-85,185$ & $-85,132$ & $-26,212$ & $-26,139$ \\
0,220 & $-91,635$ & $-91,571$ & $-29,476$ & $-29,387$ \\
0,230 & $-82,956$ & $-82,874$ & $-35,651$ & $-35,539$ \\
0,240 & $-172,922$ & $-172,734$ & $-49,334$ & $-49,188$ \\
0,250 & $-319,108$ & $-318,996$ & $-78,409$ & $-78,327$ \\
0,260 & 265,349 & 264,834 & 68,177 & 67,648 \\
\hline
\end{tabular}

Table 2. Raab and de Lange expression (RL) and alternative expression (A) for the xx component of inverse permeability. All results are reported in atomic units.

\begin{tabular}{|lcccc|}
\hline$\omega\left(\mathrm{E}_{h}\right)$ & $X_{z z}^{R L}\left(D_{y}=0\right)$ & $X_{z z}^{R L}\left(D_{y}=1\right)$ & $X_{z z}^{A}\left(D_{y}=0\right)$ & $X_{z z}^{A}\left(D_{y}=1\right)$ \\
\hline 0,001 & $-7,389$ & $-7,390$ & $-7,391$ & $-7,392$ \\
0,100 & $-6,347$ & $-6,356$ & $-6,644$ & $-6,643$ \\
0,150 & $-4,381$ & $-4,402$ & $-5,386$ & $-5,381$ \\
0,170 & $-2,796$ & $-2,826$ & $-4,495$ & $-4,486$ \\
0,180 & $-1,574$ & $-1,609$ & $-3,867$ & $-3,858$ \\
0,190 & 0,255 & 0,214 & $-2,999$ & $-2,988$ \\
0,200 & 3,391 & 3,439 & $-1,621$ & $-1,606$ \\
0,205 & 6,160 & 6,106 & $-0,515$ & $-0,499$ \\
0,210 & 10,372 & 10,315 & 1,132 & 1,149 \\
0,220 & 0,048 & 0,023 & $-2,199$ & $-2,177$ \\
0,230 & $-37,656$ & $-37,744$ & $-14,354$ & $-14,325$ \\
0,240 & $-7,626$ & $-7,822$ & $-10,605$ & $-10,649$ \\
0,250 & 14,026 & 13,904 & $-11,729$ & $-11,760$ \\
0,260 & 13,285 & 13,805 & 18,478 & 18,463 \\
\hline
\end{tabular}

The results obtained confirm the origin independence of the Raab and de Lange and of the alternative expressions, with good accuracy, and they are plotted in Figure 1. At small frequencies, the Raab and de Lange and the alternative expressions show analogues behaviour, since they both have the same static limit. In contrast, close to resonances, the Raab and de Lange expression is four times larger than the alternative expression for all components. Therefore, the resonance region is significant in order to discern between the two expressions. Note that the xx-component of the inverse permeability is always negative, whereas the zz-component and the yy-component (not showed here) change their sign at resonance frequencies.

Figure 2 analyze the dispersion behaviour of the dominant contributions in the Raab and de Lange and the alternative expressions. According to the definition of these contributions in Table 1 in terms of multipole moments, we can observe the presence of poles in accordance with the first resonance frequencies, where the 


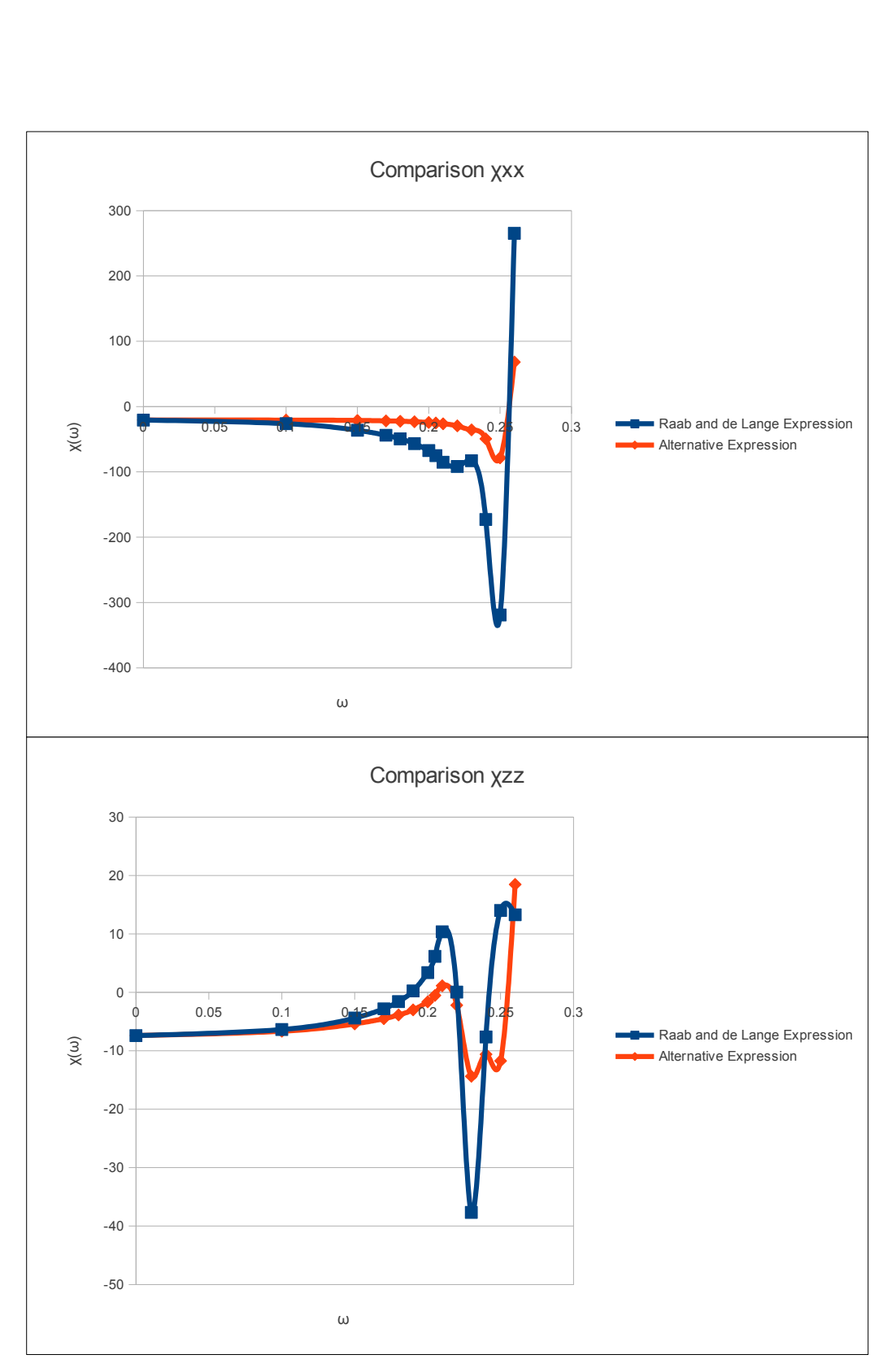

Figure 1. Comparison of the xx- and zz-component of the Raab and de Lange and the alternative expressions, varying the frequency of the external field.

\begin{abstract}
electric dipole, quadrupole, octopole and magnetic dipole, quadrupole moments are either allowed or forbidden by symmetry. The analysis of these poles explain the behaviour of both expressions showed in Figure 1. Note that the contraction and the coefficient in front of the various tensors are different in the two expressions, explaining the different values of $d$ in Figure 2 .

In the expressions for the inverse permeability, the first-order contribution is the standard magnetizability $\chi_{\imath \jmath}$, which is origin dependent by itself at non-zero frequencies. Therefore, the higher-order contributions are essential in order to recover origin independence, and they have the same order in magnitude of the standard magnetizability, as shown in figure 2 .
\end{abstract}

.

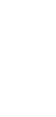

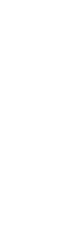

( 


\section{3. $\quad$ Electric permittivity}

Calculations of the two different expressions for the electric permittivity, given in eqs.(16) and (45), have been performed following the same approach and methods introduced and discussed in the previous section for the inverse permeability. In particular, the origin independence of these expressions and their differences in magnitude have been investigated.

Tables 4 and 5 report the results of the calculations of the Raab and de Lange and the alternative expressions. We have reported the values of the xx-component and the zz-component of the electric permittivity $\left(A_{x x}, A_{z z}\right)$ of both expressions, calculated at the center of mass and when a shift along the y-axis is considered.

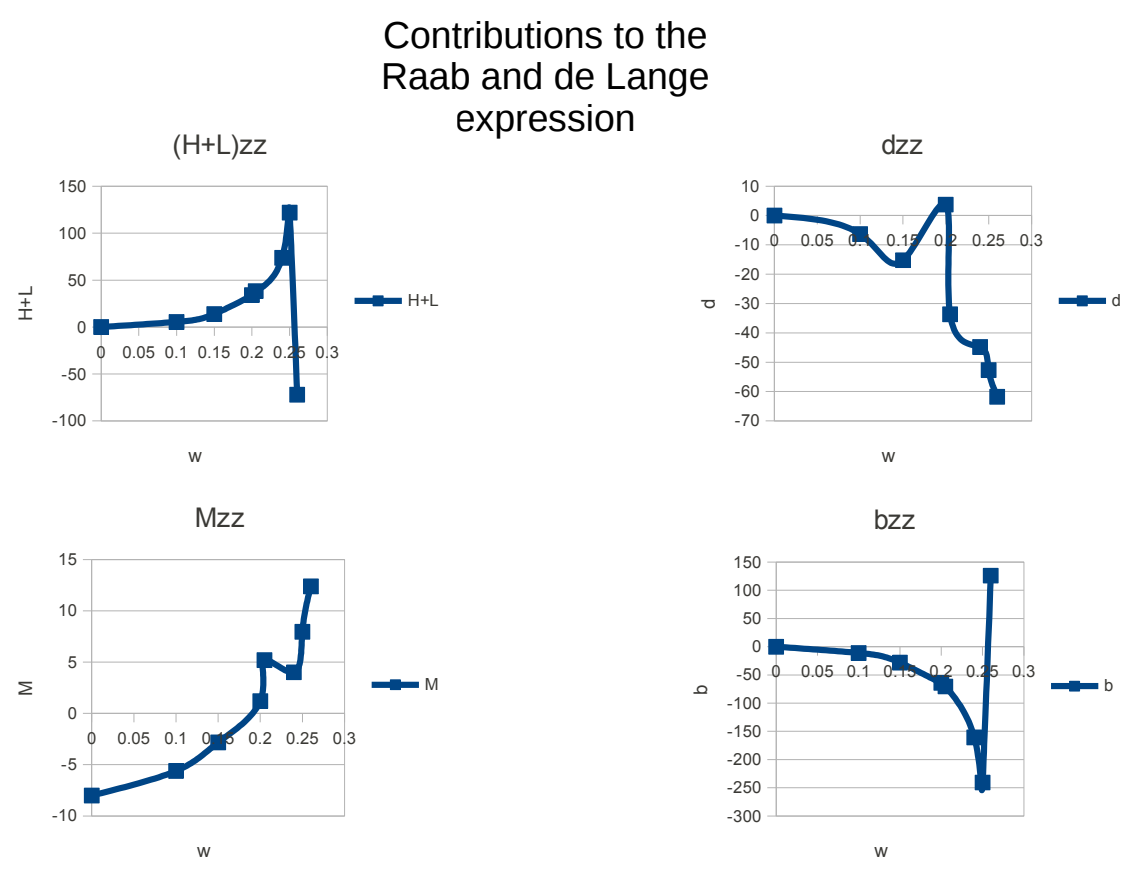

Contributions to the alternative expression
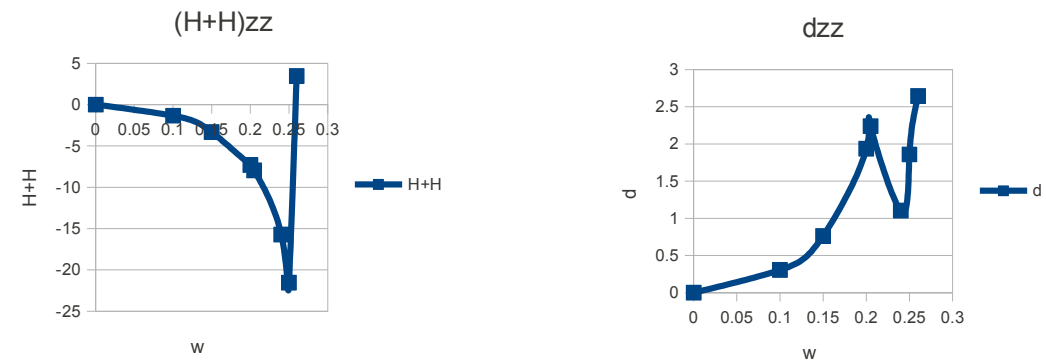

w

Figure 2. Analysis of the different contributions to the zz-component of the inverse permeability X using the Raab and de Lange and the alternative expressions, varying the frequency of the external field. All quantities are reported in atomic units. 
As expected, origin independence is seen to be recovered in all cases. We note for the permittivity that the polarizability enters in both expressions and is much larger than all other contributions. Thus the Raab and de Lange and the alternative expressions have similar values for all frequencies, as can be seen in Figure 3.

We have in Sections 3 and 4 discussed how the electric permittivity can be measured through its relation to the absorption coefficient and to the scattering amplitude. Our analysis indicates that it will be hard to distinguish between the Raab and de Lange and the alternative expressions from an experimental measurement, since they are both dominated by the polarizability and they only differ in the higher-order contributions, which are most likely too small in magnitude with respect to $\alpha_{\imath \jmath}$ to be measured. This is different to the case of the inverse permeability discussed above, where the expressions differ at resonances, and experimental measurements could be able to distinguish between the two expressions.

\begin{tabular}{|lclll|}
\hline$\omega\left(\mathrm{E}_{h}\right)$ & $A_{x x}^{R L}\left(D_{y}=0\right)$ & $A_{x x}^{R L}\left(D_{y}=1\right)$ & $A_{x x}^{A}\left(D_{y}=0\right)$ & $A_{x x}^{A}\left(D_{y}=1\right)$ \\
\hline 0,001 & 44,791 & 44,791 & 44,683 & 44,683 \\
0,100 & 46,941 & 46,941 & 46,860 & 46,860 \\
0,150 & 50,431 & 50,431 & 50,398 & 50,398 \\
0,170 & 52,760 & 52,760 & 52,761 & 52,761 \\
0,180 & 54,330 & 54,330 & 54,344 & 54,344 \\
0,190 & 56,311 & 56,311 & 56,343 & 56,343 \\
0,200 & 58,930 & 58,930 & 59,054 & 59,054 \\
0,205 & 60,607 & 60,607 & 60,777 & 60,777 \\
0,210 & 62,718 & 62,718 & 62,837 & 62,837 \\
0,220 & 691,168 & 69,168 & 69,243 & 69,243 \\
0,230 & 82,401 & 82,401 & 82,283 & 82,283 \\
0,240 & 51,732 & 51,732 & 51,840 & 51,840 \\
0,250 & 32,100 & 32,100 & 32,544 & 32,544 \\
0,260 & 45,714 & 45,715 & 45,507 & 45,507 \\
\hline
\end{tabular}

Table 4. Raab and de Lange expression (RL) and alternative expression (A) for the xx component of electric permittivity. All results are reported in atomic units.

\begin{tabular}{|lcccc|}
\hline$\omega\left(\mathrm{E}_{h}\right)$ & $A_{z z}^{R L}\left(D_{y}=0\right)$ & $A_{z z}^{R L}\left(D_{y}=1\right)$ & $A_{z z}^{A}\left(D_{y}=0\right)$ & $A_{z z}^{A}\left(D_{y}=1\right)$ \\
\hline 0,001 & 81,746 & 81,746 & 81,542 & 81,542 \\
0,100 & 88,454 & 88,453 & 88,247 & 88,247 \\
0,150 & 100,405 & 100,405 & 100,189 & 100,405 \\
0,170 & 108,876 & 108,876 & 108,876 & 108,876 \\
0,180 & 114,871 & 114,871 & 114,871 & 114,871 \\
0,190 & 122,569 & 122,569 & 122,569 & 122,569 \\
0,200 & 133,159 & 133,158 & 132,896 & 132,896 \\
0,205 & 139,714 & 139,714 & 139,439 & 139,449 \\
0,210 & 147,227 & 147,227 & 147,227 & 147,227 \\
0,220 & 168,972 & 168,972 & 168,972 & 168,972 \\
0,230 & 205,699 & 205,699 & 205,699 & 205,699 \\
0,240 & 280,282 & 280,282 & 279,714 & 279,714 \\
0,250 & 426,490 & 426,490 & 425,525 & 425,525 \\
0,260 & $-271,164$ & $-271,157$ & $-270,293$ & $-270,293$ \\
\hline
\end{tabular}

Table 5. Raab and de Lange expression (RL) and alternative expression (A) for the zz component of electric permittivity. All results are reported in atomic units. 


\section{Summary and concluding remarks}

Multipole theory provides origin-dependent expressions for the material constants in both the microscopic and the macroscopic case. Origin-independent expressions of the material constants have been derived by Raab and de Lange applying a transformed multipole theory of the auxiliary fields [7]. The uniqueness of these expressions have been discussed in this work, with special attention given to the electric permittivity and the inverse permeability.

The covariant formulation of Maxwell's equations also leads to origin-independent definitions of the material constants, which can be considered equally valid as the results of transformed multipole theory, since they both are origin independent and do not affect the spatial invariance of the wave equation.

We have recovered the expression of the electric permittivity by transformed multipole theory given in eq.(16), using different derivations based on the description of the interaction between radiation and matter [11]. In particular, the absorption coefficient and the scattering amplitude can be written in terms of the electric permittivity defined by the expression provided by Raab and De Lange. Thus, we have provided a quantum-mechanical derivation of the expression for the electric per-
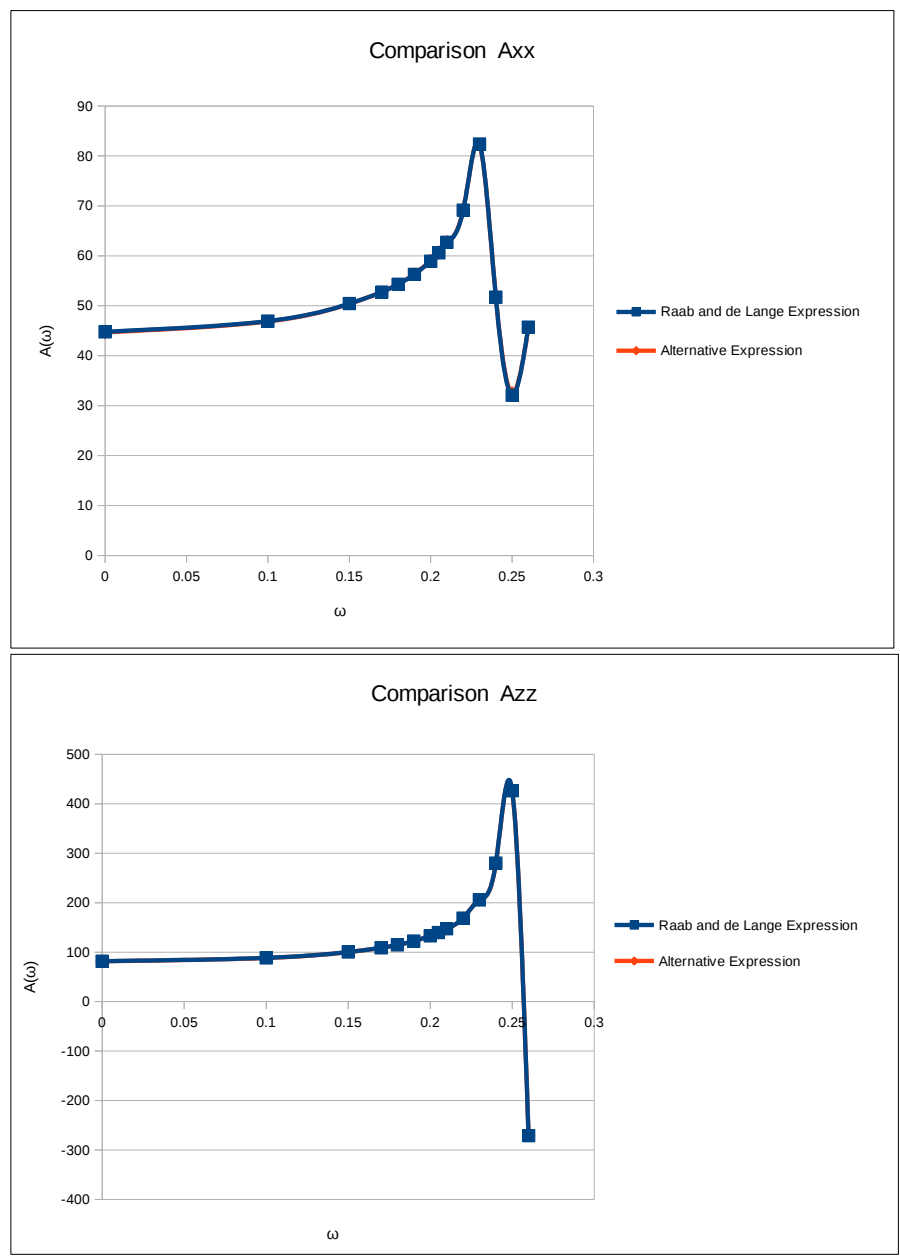

Figure 3. Axx and Azz components of the electric permittivity at the origin of the coordinates (blue) and after a shift of 1 Bohr along the y-axis (red). 
mittivity identical to the one obtained using transformed multipole theory starting from observable quantities.

The two alternative definitions of the inverse permeability given in eq.(19) and eq.(48) are both covariant, origin independent and preserve the spatial invariance of the wave equation. We have not been able to find a rigorous derivation that would allow us to prefer one expression over the other. However, considering the results obtained for the electric permittivity, which agree with the expressions provided by transformed multipole theory, support is given to eq.(19) as the definition of the inverse permeability. We thus feel that this work provides strong support for the notion that the origin-independent expressions of the material constants are provided by transformed multipole theory, as given in eqs.(16)-(19). Furthermore, we should note that in the microscopic case, an origin-independent expression for the frequency-dependent magnetizability, equivalent to the one obtained applying transformed multipole theory, has been achieved.

We have discussed the wave equation written in terms of the material constants, noting that different expressions for the material constants can be considered valid as long as the form and the spatial invariance of the wave equation is preserved. Therefore, the wave equation does not provide a constraint that could help to discern between different definitions of the material constants. This observation also explains why the origin independence of the material constants often have not been discussed in literature. In fact observable quantities that are derived from the wave equation, such as for example the refractive index, do not suffer from origin dependence, since the wave equation is origin independent, irrespective of the definition of the material constants. However, this is not the case when considering reflective properties. Our derivation of the scattering amplitude in terms of the electric permittivity also emphasizes the need for origin-independent definitions of the material constants.

We have performed calculations on the benzene molecule for various frequency values. The two different expressions for the inverse permeability and the electric permittivity provided by transformed multipole theory and by the covariant formulation of the constitutive relations have been investigated, verifying their origin independence and comparing their numerical values. Analogues results have been obtained for both expressions for the electric permittivity, which are strongly dominated by the first-order contribution (polarizability), whereas for frequencies close to resonances, the expressions of the inverse permeability show different behaviours. In particular, the Raab and de Lange expression is four times larger in magnitude with respect to the covariant expression. Therefore, we conclude that the resonant region provides useful information in order to discern between the two expressions for the inverse permeability, should it be possible to measure the dispersion curves of the inverse permeability.

\section{Appendix}

Referring to Healy's work, we here derive the result obtained in Section 3 using the expression of the scattering amplitude provided by the multipolar formulation of molecular quantum electrodynamics, written in terms of multipole integrals: 


$$
\begin{aligned}
f_{2 \leftarrow 1}= & -k_{1}^{-1 / 2} k_{2}^{3 / 2}\left\{\left(\boldsymbol{k}_{1} \wedge \boldsymbol{e}^{\left(\lambda_{1}\right)}\right)_{\imath}\left(\boldsymbol{k}_{2} \wedge \boldsymbol{e}^{\left(\lambda_{2}\right) *}\right)_{\jmath} O_{\imath \jmath}^{n_{2} n_{1}}\left(\boldsymbol{k}_{1},-\boldsymbol{k}_{2}\right)\right. \\
& +\sum_{n}\left[e_{\imath}^{\left(\lambda_{1}\right)} e_{\imath}^{\left(\lambda_{2}\right) *}\left(\frac{P_{\jmath}^{n_{2} n}\left(-\boldsymbol{k}_{2}\right) P_{\imath}^{n n_{1}}\left(\boldsymbol{k}_{1}\right)}{k_{1}-k_{n n_{1}}}-\frac{P_{\imath}^{n_{2} n}\left(\boldsymbol{k}_{1}\right) P_{\jmath}^{n n_{1}}\left(-\boldsymbol{k}_{2}\right)}{k_{2}+k_{n n_{1}}}\right)\right. \\
+ & \left(\boldsymbol{k}_{1} \wedge \boldsymbol{e}^{\left(\lambda_{1}\right)}\right)_{\imath} e_{\imath}^{\left(\lambda_{2}\right) *}\left(\frac{P_{\jmath}^{n_{2} n}\left(-\boldsymbol{k}_{2}\right) M_{\imath}^{n n_{1}}\left(\boldsymbol{k}_{1}\right)}{k_{1}-k_{n n_{1}}}-\frac{M_{\imath}^{n_{2} n}\left(\boldsymbol{k}_{1}\right) P_{\jmath}^{n n_{1}}\left(-\boldsymbol{k}_{2}\right)}{k_{2}+k_{n n_{1}}}\right) \\
+ & e_{\imath}^{\left(\lambda_{1}\right)}\left(\boldsymbol{k}_{2} \wedge \boldsymbol{e}^{\left(\lambda_{2}\right) *}\right)_{\jmath}\left(\frac{M_{\jmath}^{n_{2} n}\left(-\boldsymbol{k}_{2}\right) P_{\imath}^{n n_{1}}\left(\boldsymbol{k}_{1}\right)}{k_{1}-k_{n n_{1}}}-\frac{P_{\imath}^{n_{2} n}\left(\boldsymbol{k}_{1}\right) M_{\jmath}^{n n_{1}}\left(-\boldsymbol{k}_{2}\right)}{k_{2}+k_{n n_{1}}}\right) \\
+ & \left.\left.\left(\boldsymbol{k}_{1} \wedge \boldsymbol{e}^{\left(\lambda_{1}\right)}\right)_{\imath}\left(\boldsymbol{k}_{2} \wedge \boldsymbol{e}^{\left(\lambda_{2}\right) *}\right)_{\jmath}\left(\frac{M_{\jmath}^{n_{2} n}\left(-\boldsymbol{k}_{2}\right) M_{\imath}^{n n_{1}}\left(\boldsymbol{k}_{1}\right)}{k_{1}-k_{n n_{1}}}-\frac{M_{\imath}^{n_{2} n}\left(\boldsymbol{k}_{1}\right) M_{\jmath}^{n n_{1}}\left(-\boldsymbol{k}_{2}\right)}{k_{2}+k_{n n_{1}}}\right)\right]\right\}
\end{aligned}
$$

The quantities that appear in this expression can be identified as the matrix elements of the electric and magnetic multipole operators, defined as

$$
\begin{gathered}
O_{\imath \jmath}^{n n^{\prime}}\left(\boldsymbol{k} \boldsymbol{k}^{\prime}\right)=\left(e^{2} / m\right)\langle n|\left[(\boldsymbol{q}-\boldsymbol{R})^{2} \delta_{\imath \jmath}-(\boldsymbol{q}-\boldsymbol{R})_{\imath}(\boldsymbol{q}-\boldsymbol{R})_{\jmath}\right] \\
\int_{0}^{1} \theta e^{\imath \boldsymbol{k} \cdot[\boldsymbol{R}+\theta(\boldsymbol{q}-\boldsymbol{R})]} d \theta \int_{0}^{1} \theta^{\prime} e^{\imath \boldsymbol{k}^{\prime} \cdot\left[\boldsymbol{R}+\theta^{\prime}(\boldsymbol{q}-\boldsymbol{R})\right]} d \theta^{\prime}\left|n^{\prime}\right\rangle \\
\boldsymbol{P}^{n n^{\prime}}(\boldsymbol{k})=-e\left\langle n\left|(\boldsymbol{q}-\boldsymbol{R}) \int_{0}^{1} e^{\imath \boldsymbol{k} \cdot[\boldsymbol{R}+\theta(\boldsymbol{q}-\boldsymbol{R})]} d \theta\right| n^{\prime}\right\rangle \\
\boldsymbol{M}^{n n^{\prime}}(\boldsymbol{k})=-\frac{e}{2 m}\left\langle n\left|\left[\boldsymbol{l} \int_{0}^{1} \theta e^{\imath \boldsymbol{k} \cdot[\boldsymbol{R}+\theta(\boldsymbol{q}-\boldsymbol{R})]} d \theta+\int_{0}^{1} \theta e^{\imath \boldsymbol{k} \cdot[\boldsymbol{R}+\theta(\boldsymbol{q}-\boldsymbol{R})]} d \theta \boldsymbol{l}\right]\right| n^{\prime}\right\rangle
\end{gathered}
$$

where $\boldsymbol{R}$ is the position vector, and $\theta$ is a parameterization of the integration variable (position), the angular momentum $\boldsymbol{l}$ is defined with respect to $\boldsymbol{R}$. Healy showed [10] that eqs. (22) and (59) are equivalent if the energy conservation condition $k_{1}+k_{n_{1}}=k_{2}+k_{n_{2}}$ is preserved.

In order to re-derive the expression for the permittivity starting from the scattering amplitude, let us start by considering eq.(59), the definitions of the integrals given in eqs.(60)-(62), and the Taylor expansion up to second order of the exponential describing the external perturbation

$$
e^{\imath \theta \boldsymbol{k} \cdot \boldsymbol{q}}=1+\imath(\boldsymbol{k} \cdot \boldsymbol{q}) \theta-1 / 2(\boldsymbol{k} \cdot \boldsymbol{q})^{2} \theta^{2}+\ldots
$$

It is important to note that, without loss of generality, the center of the multipole expansion has been put as the atomic center, chosen to be located at $R=0$. Inserting this expansion in eqs.(60)-(62) and performing the integration over $\theta$ gives

$$
O_{\imath \jmath}^{n_{2} n_{1}}\left(\boldsymbol{k}_{\mathbf{1}},-\boldsymbol{k}_{2}\right)=\left(e^{2} / m\right)\left\langle n_{2}\left|\frac{1}{4}\left(\boldsymbol{q}^{2} \delta_{\imath \jmath}-q_{\imath} q_{\jmath}\right)\right| n_{1}\right\rangle
$$




$$
\begin{gathered}
P_{\imath}^{n_{2} n}(\boldsymbol{k})=-e\left\langle n_{2}\left|q_{\imath}\left(1+\frac{1}{2} \imath k_{k} q_{k}-\frac{1}{6} k_{k} k_{l} q_{k} q_{l}\right)\right| n\right\rangle \\
M_{\imath}^{n n_{1}}(\boldsymbol{k})=-\frac{e}{2 m}\left\langle n\left|\left[l_{\imath}\left(\frac{1}{2}+\frac{1}{3} \imath k_{k} q_{k}-\frac{1}{8} k_{k} k_{l} q_{k} q_{l}\right)+\left(\frac{1}{2}+\frac{1}{3} \imath k_{k} q_{k}-\frac{1}{8} k_{k} k_{l} q_{k} q_{l}\right) l_{\imath}\right]\right| n_{1}\right\rangle
\end{gathered}
$$

for each of the different terms that contribute to eq. (59), in which we can identify electric and magnetic multipole moments.

Substituting eqs.(64)-(66) into the expression for the scattering amplitude (eq.(59)), we can rewrite $f_{2 \leftarrow 1}$ as a linear combination of response functions, or alternatively as a linear combination of polarizabilities [1].

Thus, the expression for the scattering amplitude provided by the multipolar formulation of molecular quantum electrodynamics can be written as

$$
\begin{aligned}
f_{2 \leftarrow 1}= & e_{\imath}^{\left(\lambda_{1}\right)} e_{\jmath}^{\left(\lambda_{2}\right) *}\left[\alpha_{\imath \jmath}+\frac{1}{2} \imath k_{k}\left(a_{\imath \jmath k}-a_{\jmath \imath k}\right)-\frac{1}{6} k_{k} k_{l}\left(b_{\imath \jmath k l}+b_{\jmath \imath k l}\right)\right. \\
& +\frac{1}{4} d_{\imath k \jmath l}-\imath \varepsilon_{\imath k l} k_{k}\left(G_{l \jmath}^{\prime}+G_{\jmath l}^{\prime}\right)+\varepsilon_{\imath k m} \varepsilon_{\jmath l n} k_{k} k_{l} \chi_{m n} \\
& \left.-\frac{1}{2} \varepsilon_{\imath k m} k_{k} k_{l}\left(H_{\jmath m l}^{\prime}-L_{\jmath l m}^{\prime}\right)-\frac{1}{2} \varepsilon_{\jmath k m} k_{k} k_{l}\left(H_{\imath m l}^{\prime}-L_{\imath l m}^{\prime}\right)\right] \\
& =e_{\imath}^{\left(\lambda_{1}\right)} e_{\jmath}^{\left(\lambda_{2}\right) *} A_{\imath \jmath}
\end{aligned}
$$

This is equal to eq.(22) when performing a Taylor expansion of the perturbation, providing a unique definition of the permittivity being equivalent to the one achieved in transformed multipole theory (eq.(16)).

\section{Acknowledgments}

This work has received support from the Research Council of Norway through a Centre of Excellence Grant (Grant No. 179568/V30), and from the Norwegian Supercomputing Program (NOTUR) through a grant of computer time (Grant No. NN4654K). The authors would like to thank Trond Saue for many helpful discussions.

\section{References}

[1]R.E.Raab and O. Lange, Multipole theory in electromagnetism. Oxford: Clarendon, 2005.

[2] J.D.Jackson, Classical Electrodynamics. USA: John Wiley and Sons,Inc., 1962.

[3] R.E.Raab and E.B.Graham, "Universal constitutive relations for optical effects in transmission and reflection in magnetic crystals," Ferroelectrics, vol. 204, pp. 157-171, 1977.

[4]P. Visschere, "On the origin dependence of multipole moments in electromagnetism," J.Phys.D:Appl.Phys., vol. 39, pp. 4278-4283, 2006.

[5]R.E.Raab and O. Lange, "Comment on 'on the origin dependence of multipole moments in electromagnetism," J.Phys.D:Appl.Phys., vol. 43, p. 580001, 2010.

[6]P. Visschere, "Reply to comment on 'on the origin dependence of multipole moments in electromagnetism'," J.Phys.D:Appl.Phys., vol. 43, p. 5008002, 2010.

[7]R.E.Raab and O. Lange, "Transformed multipole theory of the response fields $\mathrm{d}$ and $\mathrm{h}$ to electric octopole-magnetic quadrupole," Proc.R.Soc., vol. 461, pp. 595-608, 2005.

[8]R.E.Raab and O. Lange, "On the ac magnetizability of a molecule," Molecular Physics, vol. 104, pp. 1925-1929, 2006.

[9] E.B.Graham and R.E.Raab, "Multipole solution for the macroscopic electromagnetic boundary conditions at a vacuum-dielectric interface," Proc.R.Soc.London, vol. 456, pp. 1193-1215, 2000. 
[10]W.P.Healy, "A generalization of the kramers-heisenberg dispersion formula," Phys.Rev.A, vol. 16, pp. 1568-1574, 1997.

[11]Y. J.Linderberg, Propagators in quantum chemistry. USA: John Wiley and Sons, 2004.

[12] E.J.Post, Formal structure of electromagnetism. Amsterdam: North-Holland, 1962.

[13]J. E.B.Graham and R.E.Raab, "Multipole moments and maxwell's equations," J.Phys.B, vol. 25, pp. 4673-4684, 1992.

[14] A.D.Buckingham, "Permanent and induced molecular moments and long-range intermolecular forces," Advan.Chem.Phys., vol. 12, pp. 107-142, 1967.

[15]E.B.Graham and R.E.Raab, "Light propagation in cubic and other anisotropic crystals," Proc.R.Soc.London, vol. 430, pp. 593-614, 1990.

[16] W.Heisenberg and H.A.Kramers, "On the dispersal of radiation by atoms," Zeitschrift Fur Physik, vol. 31, pp. 681-708, 1925.

[17]A. S.Bernadotte and C.R.Jacob, "Origin-independent calculation of quadrupole intensities in x-ray spectroscopy," J.Chem.Phys., vol. 137, pp. 204106-15, 2012.

[18] J. X. W. X. S.Zhang, Y.S.Park, "Negative refractive index in chiral metamaterials," Phys.Rev.Lett., vol. 102, pp. 023901-4, 2009.

[19]DALTON, a molecular electronic structure program, Release 2.0 (2005), “ http://daltonprogram.org/.."

[20]P. J. Stephens, F. J. Devlin, C. F. Chabalowski, and M. J. Frisch, "ab-initio calculation of vibrational absorption and circular-dichroism spectra using density-functional force-fields," J. Phys. Chem., vol. 98, p. 11623, 1994.

[21]T. Jr., "Gaussian-basis sets for use in correlated molecular calculations.1.the atoms boron through neon and hydrogen," J.Chem.Phys., vol. 90, pp. 1007-1023, 1989.

[22]D.E.Woon and T. Jr., "Gaussian-basis sets for use in correlated molecular calculations.4.calculation of static electrical response properties," J.Chem.Phys., vol. 100, pp. 2975-2988, 1994.

[23] K.Ruud and T.Helgaker, "The magnetizability, rotational g tensor, and quadrupole moment of pf3 revisited," Chem.Phys.Lett., vol. 264, pp. 17-23, 1997.

[24]F.London, "The quantic theory of inter-atomic currents in aromatic combinations," J.Phys.Radium, vol. 8, pp. 397-409, 1937.

[25]K. T. K. P.Jorgensen and H.J.Aa.Jensen, "Hartree-fock limit magnetizabilities from london orbitals," J.Chem.Phys., vol. 99, pp. 3847-3859, 1993.

[26]C. D.J.Wilson and T.Helgaker, "The rotational g tensor as a benchmark for density-functional theory calculations of molecular magnetic properties," J.Chem. Theory Comput., vol. 1, pp. 877-888, 2005.

[27]H. P.Norman, D.M.Bishop and J.Oddershede, "Near-resonant absorption in the time-dependent selfconsistent field and multiconfigurational self-consistent field approximations," J.Chem.Phys., vol. 115, pp. 10323-10334, 2001. 